\title{
RANDOM FIXED POINTS FOR $*$-NONEXPANSIVE RANDOM OPERATORS
}

\author{
ABDUL RAHIM KHAN ${ }^{1}$ \\ King Fahd University of Petroleum and Minerals \\ Department of Mathematical Sciences \\ Dhahran 31261, Saudi Arabia \\ NAWAB HUSSAIN \\ Bahauddin Zakariya University \\ Centre for Advanced Studies in Pure and Applied Mathematics \\ Multan 60800, Pakistan
}

(Received December, 1999; Revised March, 2001)

The notion of a $*$-nonexpansive multivalued map is different from that of a continuous map. In this paper we prove some fixed point theorems for *-nonexpansive multivalued random operators in the setup of Banach spaces and Fréchet spaces. Our work generalizes, refines and improves the earlier results of a number of authors.

Key words: Random Fixed Point, *-Nonexpansive Random Map, Banach Space, Fréchet Space, Weakly Inward Operator, Leray-Schauder Condition.

AMS subject classifications: $47 \mathrm{H} 10,54 \mathrm{H} 25,60 \mathrm{H} 25$.

\section{Introduction}

Probabilistic functional analysis is an important mathematical discipline because of its applications to probabilistic models in applied problems. Random operators lie at the heart of this discipline and their theory is needed for the study of various classes of random equations. The study of random fixed point theorems was initiated by the Prague school of probabilists in the 1950s. The generalization of these theorems from self maps to nonself maps has gained tremendous importance after the papers by Beg [2], Beg and Shahzad [3-5], Lin [12, 13], Sehgal and Singh [16], Shahzad [18] and Tan and Yuan [19, 20]. In particular, Lin [12], Shahzad [18] and Tan and Yuan [19] studied random fixed points of 1-set-contractive maps. The class of 1-set-contractive

\footnotetext{
${ }^{1}$ On leave from the Centre for Advanced Studies in Pure and Applied Mathematics, Bahauddin Zakariya University, Multan, Pakistan. 
random maps includes condensing, nonexpansive and other interesting random maps such as locally almost nonexpansive (LANE) and semicontractive random maps. The purpose of this paper is to study the random fixed point theory of *-nonexpansive multivalued operators (which are not continuous) defined on convex and star-shaped subsets of Banach spaces as well as Fréchet spaces. Recent results of Beg [2], Shahzad [18] and Tan and Yuan [19] follow as a special case from our results. An error in Theorem 2.2 of $\mathrm{Yi}$ and Zhao [24] is pointed out and corrected.

\section{Preliminaries}

Throughout this paper, $(\Omega, \mathcal{A})$ denotes a measurable space with $\mathcal{A}$ a $\sigma$-algebra of subsets of $\Omega$ unless stated otherwise. Let $X$ be a normed space (or a Fréchet space), $C$ a subset of $X, 2^{X}$ the family of all subsets of $X, K(X)$ the family of all nonempty compact subsets of $X, C K(X)$ the family of all nonempty convex, compact subsets of $X$, $W K(X)$ the family of all nonempty weakly compact subsets of $X$ and $C B(X)$ the family of all closed bounded subsets of $X$. A mapping $T: \Omega \rightarrow 2^{X}$ is called measurable if for any open subset $B$ of $X, T^{-1}(B)=\{\omega \in \Omega: T(\omega) \cap B \neq \emptyset\} \in \mathcal{A}$. A mapping $\xi: \Omega \rightarrow X$ is said to be a measurable selector (cf. [7, 10]) of a measurable mapping $T: \Omega \rightarrow 2^{X}$ if $\xi$ is measurable and for any $\omega \in \Omega, \xi(\omega) \in T(\omega)$. A mapping $T: \Omega \times C \rightarrow 2^{X}$ is said to be a random operator if for any $x \in C, T(\cdot, x)$ is measurable. A mapping $\xi: \Omega \rightarrow C$ is said to be

(i) a deterministic fixed point of $T$ if $\xi(\omega) \in T(\omega, \xi(\omega))$ for all $\omega \in \Omega$ and

(ii) a random fixed point of $T$ if $\xi$ is a measurable map such that for every $\omega \in \Omega, \xi(\omega) \in T(\omega, \xi(\omega))$.

A mapping $T: C \rightarrow 2^{X}$ is said to be

(i) upper (lower) semicontinuous if for any closed (open) subset $B$ of $X$, $T^{-1}(B)$ is closed (open); if $T$ is both upper and lower semicontinuous, then $T$ is called a continuous map,

(ii) demiclosed at 0 if the conditions $x_{n} \in C, x_{n} \rightarrow x$ weakly, $y_{n} \in T x_{n}, y_{n} \rightarrow 0$ strongly imply $0 \in T x$. A mapping $T: C \rightarrow C B(X)$ is a contraction if for any $x, y \in C, H(T x, T y) \leq k\|x-y\|$ where $H$ is the Hausdorff metric on $C B(X)$ and $0 \leq k<1$. If $k=1$, then $T$ is called a nonexpansive map.

A mapping $T: C \rightarrow X$ is called condensing if $T$ is continuous and for any bounded subset $B$ of $C$ with $\alpha(B)>0, \alpha(T(B))<\alpha(B)$, where $\alpha(B)=\inf \{\epsilon>0: B$ can be covered by a finite number of sets of diameter $\leq \epsilon\}$. The number $\alpha(B)$ is called the (set-) measure of noncompactness of $B$. If there exists $k, 0 \leq k \leq 1$, such that for each nonempty bounded subset $B$ of $C$ we have $\alpha(T(B)) \leq k \alpha(B)$, then a continuous map $T: C \rightarrow X$ is called a $k$-set-contractive map. In case $C$ is a convex subset of $X$, the map $T: C \rightarrow X$ is affine if $T(\lambda x+(1-\lambda) y)=\lambda T x+(1-\lambda) T y$ for all $x, y \in C$ and $0<\lambda<1$.

Let $(X, d)$ be a metrizable locally convex space. A ball $B_{r}(0)=\{z \in X$ : $d(z, 0) \leq r\}$ with radius $r$ and centered at 0 is said to be compressible if for every $\lambda>1$ there is $t>r$ such that $B_{t}(0) \subset \lambda B_{r}(0)$. If every ball $B_{r}(0)$ in $(X, d)$ is compressible (resp. convex), then we say that $d$ is compressible (resp. convex) (see [21]).

A mapping $T: C \rightarrow 2^{X}$ is said to be

(i) weakly nonexpansive (cf. $[8,22]$ ) if given $x \in C$ and $u_{x} \in T x$ there is a 
$u_{y} \in T y$ for each $y \in C$ such that $d\left(u_{x}, u_{y}\right) \leq d(x, y)$,

(ii) *-nonexpansive (cf. $[8,22])$ if for all $x, y \in C$ and $u_{x} \in T_{x}$ with $d\left(x, u_{x}\right)=$ $d\left(x, T_{x}\right)=\inf \left\{d(x, z): z \in T_{x}\right\}$, there exists $u_{y} \in T_{y}$ with $d\left(y, u_{y}\right)=d\left(y, T_{y}\right)$ such that $d\left(u_{x}, u_{y}\right) \leq d(x, y)$,

(iii) hemicompact if each sequence $\left\{x_{n}\right\}$ in $C$ has a convergent subsequence whenever $d\left(x_{n}, T x_{n}\right) \rightarrow 0$ as $n \rightarrow \infty$.

For the above map $T$ and each $x \in C$, we follow $\mathrm{Xu}$ [22] to define the set (possibly empty)

$$
P_{T}(x)=\left\{u_{x} \in T_{x}: d\left(x, u_{x}\right)=d\left(x, T_{x}\right)\right\}
$$

A random operator $T: \Omega \times C \rightarrow 2^{X}$ is said to

(i) be continuous (nonexpansive, hemicompact, *-nonexpansive, etc.) if for each $\omega \in \Omega, T(\omega, \cdot)$ is continuous (nonexpansive, hemicompact, *-nonexpansive, etc.),

(ii) be weakly inward if for each $\omega \in \Omega, T(\omega, x) \subset \operatorname{cl} I_{C}(x)$ for $x \in C$ where cl denotes closure and $I_{C}(x)=\{z \in X: z=x+a(y-x)$ for some $y \in C$ and $a \geq 0\}$,

(iii) satisfy the Leray-Schauder condition (in case $C$ has a nonempty interior) if there is a point $z$ in the interior of $C$ (depending on $\omega$ ) such that for each $u_{y} \in T(\omega, y)$

$$
u_{y}-z \neq m(y-z)
$$

for all $y \in \partial C$ (the boundary of $C$ ) and $m>1$.

A Banach space $X$ satisfies Opial's condition if for each $x \in X$ and each sequence $\left\{x_{n}\right\}$ converging weakly to $x, \lim _{n} \inf \left\|x_{n}-y\right\|>\liminf _{n}\left\|x_{n}-x\right\|$ holds for all $y \neq x$ in $X$.

A *-nonexpansive multivalued mapping is different from a continuous mapping as is clear from the following example.

Example 2.1: Let $X=\mathbb{R}^{2}$ be equipped with Euclidean norm and $C=\{(a, 0)$ : $\left.\frac{1}{\sqrt{2}} \leq a \leq 1\right\} \cup\{(0,0)\}$. Define $T: C \rightarrow 2^{X}$ by

$$
T(a, 0)=\left\{\begin{array}{cc}
(0,1) & \text { if } a \neq 0 \\
L=\text { the line segment }[(0,1),(1,0)] & \text { if } a=0
\end{array}\right.
$$

Then $P_{T}(a, 0)=\{(0,1)\}$ for all $(a, 0) \in C$ with $a \neq 0$ and $P_{T}(0,0)=\left(\frac{1}{2}, \frac{1}{2}\right)$.

Clearly $T$ is a $*$-nonexpansive discontinuous multifunction (cf. [15, p. 537]). Moreover, for given $x=(0,0)$ and $u_{x}=(1,0) \in T_{x}$, there does not exist $y \neq x$ in $C$ and $u_{y} \in T_{y}$ such that

$$
\left|u_{x}-u_{y}\right| \leq|x-y|
$$

Recall that for $y \neq x$ in $C, \quad u_{y}=(0,1)$ and $\left|u_{x}-u_{y}\right|=|(1,0)-(0,1)|=$ $\sqrt{2}>d(x, y)$. So $T$ is not weakly nonexpansive.

Remarks 2.2: (i) In view of Example 2.1, the statement "each *-nonexpansive map is weakly nonexpansive" in $[8$, p. 389] is not valid.

(ii) It follows from the definition of Hausdorff metric that a weakly nonexpansive map is nonexpansive. The converse holds for compact-valued maps. For if $T: C \rightarrow 2^{X}$ 
is a compact-valued nonexpansive map, then for any $x \in C$ and $u_{x} \in T_{x}$, we can find some $u_{y} \in T_{y}$ for all $y$ in $C$ by compactness of $T_{y}$ such that

$$
d\left(u_{x}, u_{y}\right) \leq \sup \left\{d\left(u, T_{y}\right): u \in T_{x}\right\} \leq H\left(T_{x}, T_{y}\right) \leq d(x, y) .
$$

So $T$ is weakly nonexpansive (also see Proposition 1 [22]).

(iii) *-nonexpansiveness and nonexpansiveness are two different concepts for multivalued mappings.

\section{Random Fixed Points in Banach Spaces}

A general fixed point theorem for a class of discontinuous multivalued random operators is established in the following.

Theorem 3.1: Let $C$ be a nonempty closed, bounded, convex, separable subset with nonempty interior of a strictly-convex, reflexive Banach space $X$ satisfying Opial's condition. Suppose that $T: \Omega \times C \rightarrow 2^{X}$ is a closed, convex-valued, *-nonexpansive random operator that either

(i) is weakly inward or

(ii) satisfies the Leray-Schauder condition. If $P_{T}$ is a random operator, then $T$ has a random fixed point.

Proof: Suppose that assumption $(i)$ is satisfied. As $X$ is strictly convex so each $T(\omega, x)$ is a Chebyshev set. Therefore for all $\omega \in \Omega$ and all $x \in C$,

$$
\left\{u_{x}\right\}=P_{T}(\omega, x) \in T(\omega, x)
$$

Also for each $\omega \in \Omega$ and each $x, y \in C$,

$$
d\left(P_{T}(\omega, x), P_{T}(\omega, y)\right)=d\left(u_{x}, u_{y}\right) \leq d(x, y) .
$$

This implies that $P_{T}: \Omega \times C \rightarrow X$ is a nonexpansive random operator. Further, $P_{T}(\omega, x) \in T(\omega, x) \subset \operatorname{cl}\left(I_{C}(x)\right)$ for all $x \in C$ and any $\omega \in \Omega$ so it follows that $P_{T}$ is weakly inward. We can easily show as in the proof of Theorems 3.1, 3.2 [11] that $I-P_{T}(\omega, \cdot)$ is demiclosed at 0 for each $\omega \in \Omega$. By Theorem 3.1 [2], $P_{T}$ has a random fixed point. That is, there is a measurable map $\xi: \Omega \rightarrow C$ such that

$$
\xi(\omega)=P_{T}(\omega, \xi(\omega)) \text { for all } \omega \in \Omega \text {. }
$$

Since $P_{T}(\omega, x) \in T(\omega, x)$ for all $\omega \in \Omega$ and for all $x \in C$, it follows that $\xi(\omega) \in$ $T(\omega, \xi(\omega))$ for all $\omega \in \Omega$, as desired.

Suppose that assumption (ii) holds. By the arguments used in case $(i), P_{T}$ : $\Omega \times C \rightarrow X$ is a nonexpansive random operator such that $I-P_{T}(\omega, \cdot)$ is demiclosed at 0 for each $\omega \in \Omega$. Since $P_{T}(\omega, y) \in T(\omega, y)$ for all $\omega \in \Omega$ and for all $y \in \partial C$ and $T$ satisfies the Leray-Schauder condition, it follows from (1) that $P_{T}$ also satisfies the Leray-Schauder condition. By Theorem $3.3[2], P_{T}$ and hence $T$ has a random fixed point.

Remarks 3.2: (i) Since *-nonexpansive multivalued maps are not necessarily continuous, Theorem 3.1 cannot be implied by the results of Beg and Shahzad in [3-5] 
and Tan and Yuan in [20].

(ii) If $I-T(\omega, \cdot)$ is demiclosed at 0 for each $\omega \in \Omega$ and $P_{T}$ is weakly continuous in Theorem 3.1, then $I-P_{T}(\omega, \cdot)$ is demiclosed at 0 as follows:

Let $x_{n} \rightarrow x_{0}$ weakly and $I-P_{T}(\omega, \cdot)\left(x_{n}\right) \rightarrow 0$ strongly for any $\omega \in \Omega$. Now $I-$ $P_{T}(\omega, \cdot)\left(x_{n}\right) \in I-T(\omega, \cdot)\left(x_{n}\right)$ and $I-T(\omega, \cdot)$ is demiclosed at 0 so $0 \in I-$ $T(\omega, \cdot)(x)$. Since $P_{T}: \Omega \times C \rightarrow X$ is weakly continuous so $I-P_{T}(\omega, \cdot)\left(x_{n}\right) \rightarrow x_{0}-$ $P_{T}\left(\omega, x_{0}\right)$ weakly. Also $I-P_{T}(\omega, \cdot)\left(x_{n}\right) \rightarrow 0$ weakly. Hence by the Hausdorff property of weak topology, we have that $0=I-P_{T}(\omega, \cdot)\left(x_{0}\right)$.

(iii) A continuous affine map is weakly continuous.

From Theorem 3.1, we now obtain:

Theorem 3.3: Let $C$ be a nonempty closed, bounded, convex, separable subset with nonempty interior of a strictly-convex, reflexive Banach space $X$. Suppose that $T$ : $\Omega \times C \rightarrow 2^{X}$ is a closed, convex-valued, *-nonexpansive random operator that either

(i) is weakly inward or

(ii) satisfies the Leray-Schauder condition.

If $I-T(\omega, \cdot)$ is demiclosed at 0 for each $\omega \in \Omega$ and $P_{T}$ is an affine random operator, then $T$ has a random fixed point.

We observe that if $X$ is a uniformly convex space in Theorem 3.1, then the theorem holds (with the same proof) because in this case, the demiclosedness of $I-P_{T}(\omega, \cdot)$ follows as in Browder's Theorem $3[6]$. Consequently, we get the following corollary which extends Theorem 2.6 of Itoh [9], Theorem (6) (ii) of Lin [13] and Theorem 4 of $\mathrm{Xu}$ [23].

Corollary 3.4: [18, Corollary 3.4] Let $X$ be a uniformly-convex Banach space and let $C$ (with nonempty interior) be a nonempty closed, bounded, convex, separable subset of $X$. If $T: \Omega \times C \rightarrow X$ is a nonexpansive random operator that either

(i) is weakly inward or

(ii) satisfies the Leray-Schauder condition, then $T$ has a random fixed point.

The following simple example contradicts the validity of Theorem 2.2 and hence Theorem 3.2 in [24].

Example 3.5: Let $X$ be th set of real numbers with the usual metric and $C=\{0,1\}$. Define $T: C \rightarrow X$ by $T(0)=1$ and $T(1)=0$. Then $T$ is nonexpansive, weakly inward and $(I-T)(C)=\{-1,1\}$ is a closed set. But $T$ has no fixed point. Similarly we may obtain that $T$ has no random fixed point where $T$ is defined on a suitable subset of a complete $\sigma$-finite measure space $(\Omega, \mathcal{A}, \mu)$.

We assume in the remainder of this section that $(\Omega, \mathcal{A}, \mu)$ is a complete $\sigma$-finite measure space.

A combination of some sort of convexity in $C$, Theorem 2.1 and Lemma 3.1 (due to $\mathrm{Yi}$ and Zhao [24]) provide the following affirmative result.

Theorem 3.6: Let $C$ be a nonempty closed, star-shaped subset of a separable Banach space $X$ and let $T: \Omega \times C \rightarrow K(X)$ be a weakly inward nonexpansive random operator. If for each $\omega \in \Omega, T(\omega, C)$ is bounded and $(I-T)(\omega, C)$ is closed, then $T$ has a random fixed point.

We will consider a weaker assumption on $C$ in the next result to establish an extension of Theorem 3.6 for $*$-nonexpansive random operators.

Theorem 3.7: Let $C$ be a nonempty, weakly-closed, star-shaped subset of a separable Banach space $X$ which satisfies Opial's condition and let $T: \Omega \times C \rightarrow K(X)$ 
be a weakly inward *-nonexpansive random operator such that for each $\omega \in \Omega$, $T(\omega, C) \subseteq B$ for some weakly compact subset $B$ of $X$. If $P_{T}$ is a random operator, then $T$ has a random fixed point.

Proof: As before, $P_{T}: \Omega \times C \rightarrow 2^{X}$ is a compact-valued, weakly inward nonexpansive random map (see also proof of Theorem 2 [22]). We shall show that for each $\omega \in \Omega,\left(I-P_{T}\right)(\omega, \cdot)(C)$ is closed. Let $\omega \in \Omega$ be fixed and $y$ be a limit point of $I-$ $P_{T}(\omega, \cdot)(C)$. Then there is a sequence $\left\{y_{n}\right\}$ with $y_{n} \in\left(I-P_{T}\right)\left(\omega, x_{n}\right)$ for some $x_{n} \in$ $C$ and $y_{n} \rightarrow y$. Hence $x_{n}-y_{n} \in P_{T}\left(\omega, x_{n}\right)$ and $y_{n} \rightarrow y$. Since $\left\{x_{n}-y_{n}\right\} \in P_{T}\left(\omega, x_{n}\right) \subseteq$ $T\left(\omega, x_{n}\right) \subseteq B$, there is $b \in B$ and a subsequence $\left\{x_{m}-y_{m}\right\}$ of $\left\{x_{n}-y_{n}\right\}$ such that $x_{m}-y_{m} \rightarrow b$ weakly. As $y_{m} \rightarrow y$ weakly, it follows that $x_{m} \rightarrow y-b$ weakly. Let $z=y-b$. As $C$ is weakly-closed, $z \in C$. Without loss of generality, we may assume that $x_{n}$ converges weakly to $z$. By Remarks 2.2 (ii), a compact-valued, nonexpansive map is weakly nonexpansive and so we obtain that $P_{T}$ is weakly nonexpansive. Hence for each $x_{n}-y_{n} \in P_{T}\left(\omega, x_{n}\right)$, there is a $z_{n} \in P_{T}(\omega, z)$ such that

$$
\left\|x_{n}-y_{n}-z_{n}\right\| \leq\left\|x_{n}-z\right\| \text {. }
$$

The set $P_{T}(\omega, z)$ is compact so $z_{n} \rightarrow u \in P_{T}(\omega, z)$ and $y_{n}+z_{n} \rightarrow y+u$.

It now follows from (2) that

$$
\underline{\lim }\left\|x_{n}-y-u\right\| \leq \underline{\lim }\left\|x_{n}-z\right\| .
$$

Hence by Opial's condition, we have $y+u=z$ and so $y=z-u \in\left(I-P_{T}\right)(\omega, \cdot)(C)$ for each $\omega \in \Omega$, as desired. Thus by Theorem 3.6, $P_{T}$ and hence $T$ has a random fixed point.

For single-valued maps, the concepts of *-nonexpansive and nonexpansive coincide. Hence the following two results generalize Corollary 3.5 of Tan and Yuan [19] to weakly-closed and star-shaped sets in the context of Opial spaces.

Corollary 3.8: Let $C$ be a nonempty, weakly-closed, star-shaped subset of a separable Banach space $X$ satisfying Opial's condition and let $T: \Omega \times C \rightarrow X$ be a nonexpansive random operator such that for each $\omega \in \Omega, T(\omega, C) \subseteq B$ for some weakly compact subset $B$ of $X$. If $T$ is weakly inward, then $T$ has a random fixed point.

Corollary 3.9: $\quad$ Let $C$ be a nonempty, weakly-compact, star-shaped subset of a separable Banach space $X$ satisfying Opial's condition and let $T: \Omega \times C \rightarrow C$ be a nonexpansive random operator. Then $T$ has a random fixed point.

We remark that in Corollary 3.9, the conditions (the fixed point property of $C$, the convexity of $C$ and strict convexity of $X$ needed in Theorem 1 by $\mathrm{Xu}$ [23]) are relaxed.

\section{Random Fixed Points in Fréchet Spaces}

Fixed point results in the context of Fréchet spaces have been studied in [14, 17]. In this section, we prove fixed point theorems for $*$-nonexpansive operators defined on a subset of a Fréchet space.

We shall need the following results.

Theorem A: [17, Theorem 3.3] Let $C$ be a nonempty, weakly-compact, convex subset of a separable Fréchet space $X$ and let $T: \Omega \times C \rightarrow X$ be 1-set contractive 
random operator that either

(i) is weakly inward

or

(ii) satisfies the Leray-Schauder condition.

If for any $\omega \in \Omega, T(\omega, C)$ is bounded and $I-T(\omega, \cdot)$ is demiclosed at 0 , then $T$ has a random fixed point.

Theorem B: [21, Theorem 2.1] Let $(X, d)$ be a locally convex, metrizable topological vector space with $d$ as convex and compressible metric. Then every weak sequentially compact subset $K$ of $X$ is proximinal.

Theorem C: [1, Theorem 2] Every convex proximinal set in a strictly-convex metric linear space is Chebyshev.

The following result extends Theorem 3.1 from Banach spaces to Fréchet spaces.

Theorem 4.1: Let $C$ be a nonempty, closed, bounded, convex subset with nonempty interior of a uniformly-convex separable Fréchet space $X$ and let $T: \Omega \times C \rightarrow 2^{X}$ be a closed, convex-valued, *-nonexpansive random operator that either

(i) is weakly inward

or

(ii) satisfies the Leray-Schauder condition.

If for each $\omega \in \Omega, I-T(\omega, \cdot)$ is demiclosed at 0 and $P_{T}$ is an affine random operator, then $T$ has a random fixed point.

Proof: It is well known that a closed, convex subset of a uniformly-convex Fréchet space is Chebyshev (see [1, Corollary 3]). So we may obtain as in the proof of Theorem 3.1 that $P_{T}: \Omega \times C \rightarrow X$ is a nonexpansive random operator and $I-P_{T}(\omega, \cdot)$ is demiclosed at 0 for each $\omega \in \Omega$. Moreover, the class of 1 -set contractive operators includes nonexpansive operators. By Theorem $\mathrm{A}, P_{T}$ and hence $T$ has a random fixed point.

Corollary 4.2: [19, Corollary 3.5] Let $C$ be a nonempty, weakly-compact, convex subset of a separable, uniformly-convex Banach space $X$ and let $T: \Omega \times C \rightarrow X$ be a nonexpansive random operator. If $T$ is weakly inward, then $T$ has a random fixed point.

An extension of Theorem 4.1 to the case of strictly-convex Fréchet spaces is obtained in the following.

Theorem 4.3: Let $C$ be a nonempty, weakly-compact, convex subset with nonempty interior of a strictly-convex separable Fréchet space $X$ with convex and compressible metric $d$ and let $T: \Omega \times C \rightarrow 2^{X}$ be a weakly sequentially compact, convex-valued, *nonexpansive random operator that either

(i) is weakly inward or

(ii) satisfies the Leray-Schauder condition.

If for each $\omega \in \Omega, I-T(\omega, \cdot)$ is demiclosed at 0 and $P_{T}$ is affine random operator, then $T$ has a random fixed point.

Proof: Theorems B and C imply that each $T(\omega, x)$ is Chebyshev. Thus, as before, $P_{T}: \Omega \times C \rightarrow X$ is a nonexpansive random operator that either

(i) is weakly inward or

(ii) satisfies the Leray-Schauder condition.

Moreover, as in Remarks $3.2(i i)$, we obtain that $I-P_{T}(\omega, \cdot)$ is demiclosed at 0 for each $\omega \in \Omega$. The conclusion follows from Theorem A.

A related random fixed point theorem for hemicompact operators is given below 
(see Theorems 4.8 and 4.9 in [19]).

Theorem 4.4: Let $C$ be a nonempty, closed, separable subset of a Fréchet space $X$ with convex and compressible metric d. Suppose $T: \Omega \times C \rightarrow 2^{X}$ is a weakly sequentially, compact-valued, *-nonexpansive hemicompact random operator. If for each $\omega \in \Omega, G(\omega)=\{x \in C: x \in T(\omega, x)\}$ is nonempty and $P_{T}$ is a random operator, then $T$ has a random fixed point.

Proof: As before, $P_{T}: \Omega \times C \rightarrow 2^{X}$ is a nonexpansive random operator. We show that $P_{T}$ is hemicompact. Let $\left\{x_{n}\right\}$ be any sequence in $C$ such that $d\left(x_{n}, P_{T}\left(x_{n}\right)\right) \rightarrow 0$ as $n \rightarrow \infty$. By definition of $P_{T}$, we have

$$
d\left(x_{n}, P_{T}\left(\omega, x_{n}\right)\right) \leq d\left(x_{n}, u_{x_{n}}\right)=d\left(x_{n}, T x_{n}\right) \leq d\left(x_{n}, P_{T}\left(\omega, x_{n}\right)\right) .
$$

So $d\left(x_{n}, T_{x_{n}}\right) \rightarrow 0$ as $n \rightarrow \infty$. The hemicompactness of $T$ implies that $\left\{x_{n}\right\}$ has a convergent subsequence. Hence $P_{T}$ is hemicompact. From (3) it follows that $x$ is a fixed point of $T(\omega, \cdot)$ iff $x$ is a fixed point of $P_{T}(\omega, \cdot)$. Thus $F(\omega)=\{x \in C$ : $\left.x \in P_{T}(\omega, x)\right\} \neq \emptyset$ for each $\omega \in \Omega$. By Theorem 3.1 [3] or Theorem $2.3[20], P_{T}$ and therefore $T$ has a random fixed point.

\section{Acknowledgements:}

The author, A.R. Khan, gratefully acknowledges support provided by the King Fahd University of Petroleum and Minerals during this research. The authors wish to thank Professor Ismat Beg for valuable comments and helpful suggestions.

\section{References}

[1] Ahuja, G.C., Narang, T.D. and Trehan, S., Best approximation on convex sets in metric linear spaces, Math. Nachr. 78 (1977), 125-130.

[2] Beg, I., Random fixed points of non-self maps and random approximations, $J$. Appl. Math. Stoch. Anal. 10:2 (1997), 127-130.

[3] Beg, I. and Shahzad, N., A general fixed point theorem for a class of continuous random operators, New Zealand J. Math 26:1 (1997), 21-24.

[4] Beg, I. and Shahzad, N., Random fixed point of weakly inward operators in conical shells, J. Appl. Math. Stoch. Anal. 8:3 (1995), 261-264.

[5] Beg, I. and Shahzad, N., Random fixed point theorems for multivalued inward random operators on Banach spaces, Adv. Math. Sci. Appl. 5:1 (1995), 31-37.

[6] Browder, F.E., Semicontractive and semiaccretive nonlinear mappings in Banach spaces, Bull. Amer. Math. Soc. 74 (1968), 660-665.

[7] Himmelberg, C.J., Measurable relations, Fund. Math. 87 (1975), 53-72.

[8] Husain, T. and Latif, A., Fixed points of multivalued nonexpansive maps, Math. Japon 33:3 (1988), 385-391.

[9] Itoh, S., Random fixed point theorems with an application to random differential equations in Banach spaces, J. Math. Anal. Appl. 67:2 (1979), 261-273.

[10] Kuratowski, K. and Ryll-Nardzewski, C., A general theorem on selectors, Bull. Acad. Polon. Sci. Sér. Sci. Math. Astron. Phys. 13 (1965), 397-403.

[11] Lami Dozo, E., Multivalued nonexpansive mappings and Opial's condition, 
Proc. Amer. Math. Soc. 38 (1973), 286-292.

[12] Lin, T.C., Random approximations and random fixed point theorems for continuous 1-set contractive random maps, Proc. Amer. Math. Soc. 123:4 (1995), 1167-1176.

[13] Lin, T.C., Random approximations and random fixed point theorems for nonself maps, Proc. Amer. Math. Soc. 103:4 (1988), 1129-1135.

[14] Polewczak, J., Ordinary differential equations on closed subsets of Fréchet spaces with applications to fixed point theorems, Proc. Amer. Math. Soc. 107:4 (1989), 1005-1012.

[15] Sehgal, V.M. and Singh, S.P., A generalization to multifunctions of Fan's best approximation theorem, Proc. Amer. Math. Soc. 102:3 (1988), 534-537.

[16] Sehgal, V.M. and Singh, S.P., On random approximation and a random fixed point theorem for set valued mappings, Proc. Amer. Math. Soc. 95 (1985), 9194.

[17] Shahzad, N, and Khan, L.A., Random fixed points of 1-set contractive random maps in Fréchet spaces, J. Math. Anal. Appl. 231:1 (1998), 68-75.

[18] Shahzad, N., Random fixed point theorems for various classes of 1-setcontractive maps in Banach spaces, J. Math. Anal. Appl. 203:3 (1996), 712-718.

[19] Tan, K.K. and Yuan, X.Z., Random fixed point theorems and approximation, Stoch. Anal. Appl. 15:1 (1997), 103-123.

[20] Tan, K.K. and Yuan, X.Z., Random fixed point theorems and approximation in cones, J. Math. Anal. Appl. 185 (1994), 378-390.

[21] Thaheem, A.B., Existence of best approximations, Port. Math. 42 (1983-84), 435-440.

[22] Xu, H.K., On weakly nonexpansive and *-nonexpansive multivalued mappings, Math. Japon 36:3 (1991), 441-445.

[23] Xu, H.K., Some random fixed point theorems for condensing and nonexpansive operators, Proc. Amer. Math. Soc. 110:2 (1990), 395-400.

[24] Yi, H.W. and Zhao, Y.C., Fixed point theorems for weakly inward multivalued mappings and their randomizations, J. Math. Anal. Appl. 183:3 (1994), 613-619. 


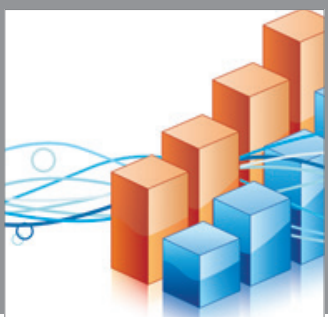

Advances in

Operations Research

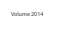

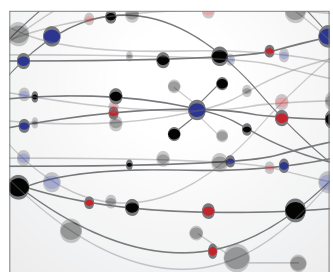

\section{The Scientific} World Journal
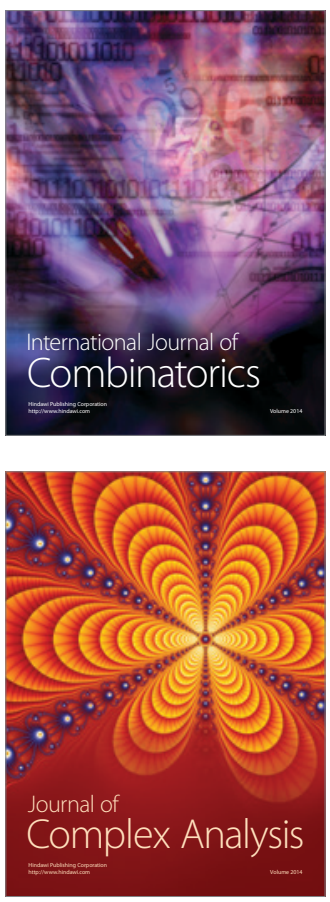

International Journal of

Mathematics and

Mathematical

Sciences
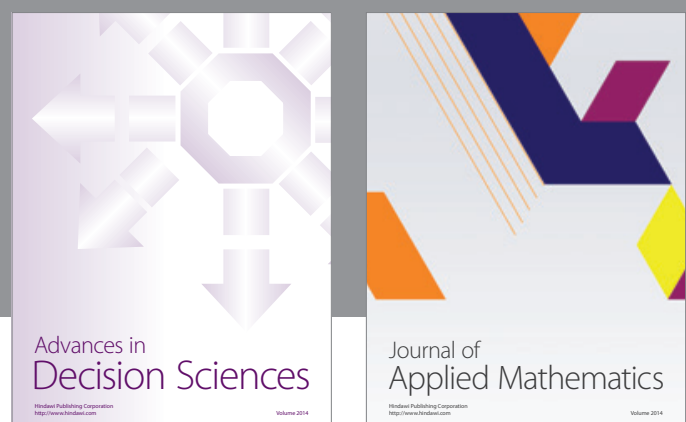

Journal of

Applied Mathematics
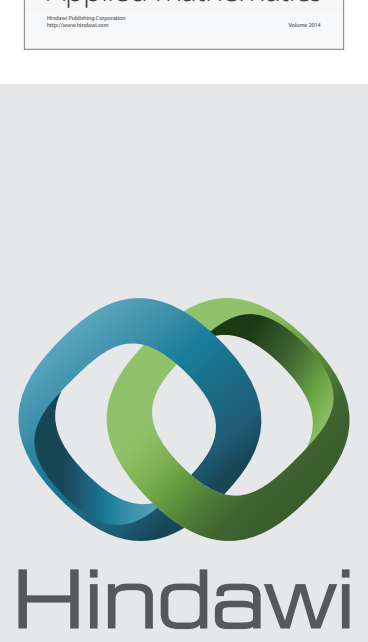

Submit your manuscripts at http://www.hindawi.com
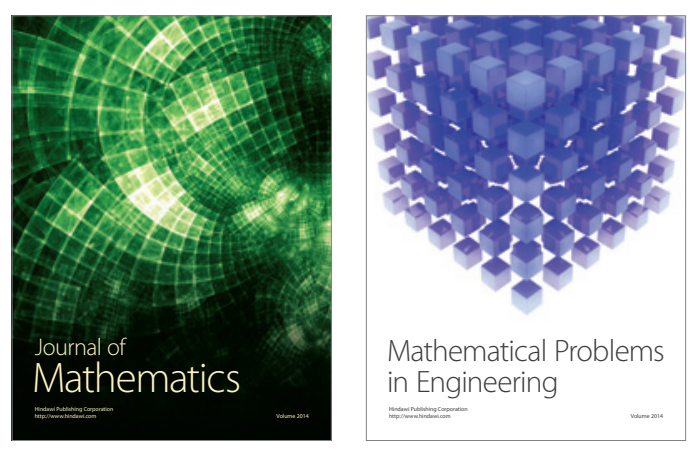

Mathematical Problems in Engineering
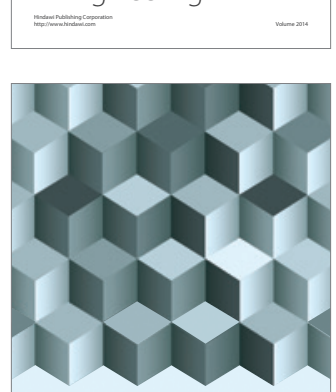

Journal of

Function Spaces
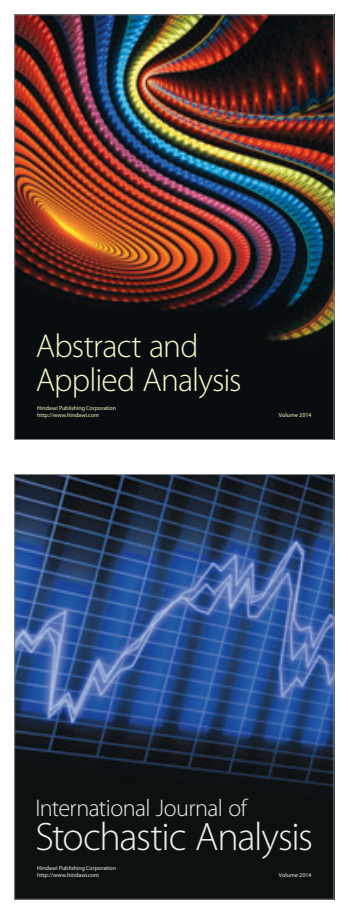

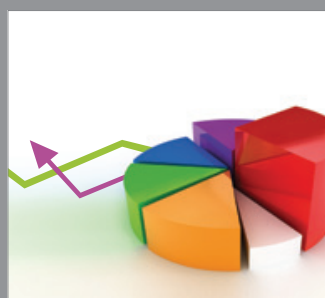

ournal of

Probability and Statistics

Promensencen
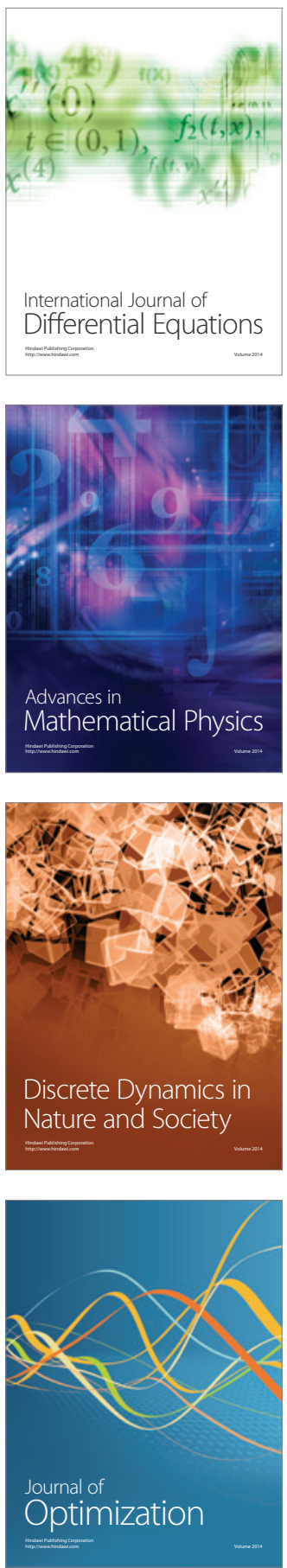